\title{
COVID-19, a tale of two peaks: patients' characteristics, treatments, and clinical outcomes
}

\author{
Ariel Banai ${ }^{1}$. Philippe Taieb ${ }^{1} \cdot$ Nadav Furie $^{2} \cdot$ Aviram Hochstadt $^{1} \cdot$ Ilan Merdler $^{1} \cdot$ Orly Sapir $^{1} \cdot$ Yoav Granot $^{1}$. \\ Lior Lupu ${ }^{1}$ - Eihab Ghantous ${ }^{1}$ - Ariel Borohovitz ${ }^{1} \cdot$ Amir Gal-Oz $^{1}$ - Merav Ingbir ${ }^{1}$ - Yaron Arbel ${ }^{1} \cdot$ Shmuel Banai ${ }^{1}$. \\ Yan Topilsky ${ }^{1} \cdot$ Yael Lichter $^{1} \cdot$ Yishay Szekely $^{1,3}$
}

Received: 21 November 2020 / Accepted: 21 January 2021 / Published online: 1 April 2021

(c) Società Italiana di Medicina Interna (SIMI) 2021

\begin{abstract}
Coronavirus 2019 disease (COVID-19) continues to challenge healthcare systems globally as many countries are currently experiencing an increase in the morbidity and mortality. Compare baseline characteristics, clinical presentation, treatments, and clinical outcomes of patients admitted during the second peak to those admitted during the first peak. Retrospective analysis of 258 COVID-19 patients consecutively admitted to the Tel Aviv Medical Center, of which, 131 during the first peak (March 21-May 30, 2020) and 127 during the second peak (May 31-July 16, 2020). First and second peak patients did not differ in baseline characteristics and clinical presentation at admission. Treatment with dexamethasone, full-dose anticoagulation, tocilizumab, remdesivir, and convalescent plasma transfusion were significantly more frequent during the second peak, as well as regimens combining 3-4 COVID-19-directed drugs. Compared to the first peak, 30-day mortality and invasive mechanical ventilation rates as well as adjusted risk were significantly lower during the second peak $(10.2 \%$, vs $19.8 \%$ vs $p=0.028$, adjusted HR $0.39,95 \%$ CI $0.19-0.79, p=0.009$ and $8.8 \%$ vs $19.3 \%, p=0.002$, adjusted HR 0.29 , $95 \%$ CI $0.13-0.64, p=0.002$; respectively). Rates of 30-day mortality and invasive mechanical ventilation, as well as adjusted risks, were lower in the second peak of the COVID-19 pandemic among hospitalized patients. The change in treatment strategy and the experienced gained during the first peak may have contributed to the improved outcomes.
\end{abstract}

Keywords COVID-19 $\cdot$ Treatments $\cdot$ Mortality $\cdot$ Mechanical ventilation

\section{Introduction}

The Coronavirus Disease 2019 (COVID-19) epidemic emerged in Wuhan in late December 2019 [1] and spread globally within weeks. By January 30, 2020, the World Health Organization (WHO) declared that COVID-19 is "a public-health emergency of international concern" [2],

Ariel Banai and Philippe Taieb contributed equally to this work.

Yishay Szekely

yishays@tlvmc.gov.il

1 Tel-Aviv Sourasky Medical Center, Sackler Faculty of Medicine, Tel-Aviv University, Tel-Aviv, Israel

2 Sheba Medical Center, Tel Hashomer, Sackler Faculty of Medicine, Tel Aviv University, Tel Aviv, Israel

3 Department of Cardiology, Tel Aviv Sourasky Medical Center, 6 Weizmann St., 64239 Tel Aviv, Israel and in March, with up to 143 countries reporting cases of COVID-19, the WHO declared the disease a pandemic [3].

In Israel, the first COVID-19 patient was diagnosed on February 21, 2020, and was soon followed by an exponential growth rate, leading to a peak of daily confirmed cases of 932 by March 25, 2020. Due to a variety of preliminary measures-isolation and quarantine, population testing, epidemiological investigations, social distancing, and public education - by early May, the rate of new daily confirmed infections plunged to 20 cases per day. However, following the withdrawal of the restrictive measures, a second peak commenced, soon surpassing the infection rate of the first peak, reaching approximately 2000 confirmed cases daily [4] (Figure S1).

The aim of the study is to compare baseline characteristics, disease severity, treatments, and clinical outcomes of patients admitted during the second peak to those admitted during the first peak. 


\section{Methods}

We retrospectively studied 258 patients with COVID-19 infection consecutively admitted to the Tel Aviv Medical Center. Patients admitted between March 21 and May 30,2020 were considered first peak patients, while those admitted between May 31 and July 16, 2020 were considered second peak patients. The start of the second peak was defined by the first increase in the rolling 7-day average daily admissions rate, which was followed by further increase. All patients had a positive reverse-transcriptasepolymerase-chain-reaction (PCR) assay for SARS-CoV-2 obtained from respiratory tract sample and were admitted to designated Internal Medicine wards or Intensive Care Units. Demographic status, comorbidities, chronic medical therapy, vital signs, physical examination findings, laboratory results, and chest X-ray findings were recorded from all patients. Upon admission, risk of clinical deterioration was evaluated using the COVID-19 modified early warning score (COVID-19 MEWS) [5] and the Sequential Organ Failure Assessment (SOFA) score [6]. Disease severity was determined in accordance with the National Institutes of Health (NIH) guidelines [7]. Components of the COVID-19 MEWS and the NIH criteria of disease severity are summarized in Supplementary Tables S1, S2, and S3. The study was approved by the Tel Aviv medical center ethics committee (IRB number 0196-20-TLV).

\section{Echocardiography and lung ultrasound}

Since the beginning of the COVID-19 pandemic, we have performed echocardiography and lung ultrasound on admission for all patients presenting with respiratory illness due to COVID-19 infection, using a pre-defined step-by-step protocol, as part of a routine patient care protocol. All patients underwent comprehensive lung ultrasound combined with bedside echocardiography within $24 \mathrm{~h}$ of admission. Echocardiographic and lung ultrasound examinations were performed by cardiologists with expertise in echocardiographic and lung ultrasound recording and interpretation. Necessary precautions were taken to minimize the risk of infection and disease transmission, in accordance with current guidelines [8]. Measurements of left-ventricular diameters, volumes, and ejection fraction (LVEF) were taken as recommended [9]. Left atrial (LA) volume was calculated using the biplane area length method at end systole. Forward stroke volume was calculated from the LV outflow tract followed by calculation of cardiac output (CO) and CO index. Right ventricular evaluation consisted of end-systolic and end-diastolic areas. Additionally, right-ventricular function was assessed by tricuspid annular plane systolic excursion (TAPSE), systolic tricuspid lateral annular velocity (RV S'), and fractional area change $[9,10]$.

Lung ultrasound examination included visualization of 6 "windows" on each side: superior and inferior anterior, superior and inferior antero-lateral, and superior and inferior postero-lateral. We used the following scoring system to rate the degree of lung aeration: A-lines, (normal repetitive reverberation of the pleura accompanied by lung sliding) received a score of 0 . B-lines (long bands of hyperechoic artifacts representing edematous interlobular septa and/or alveoli) received one or two points, depending on weather the B-lines were separated or coalescent, respectively. Lung consolidation received three points. Ultimately, the lung ultrasound score ranged from 0 (indicating normal lung) to 36 (indicating diffused bilateral lung consolidations) [11].

\section{Outcomes}

The primary outcomes were 30-day mortality and 30-day invasive mechanical ventilation. Secondary outcomes included non-invasive high flow oxygen ventilation, time to invasive mechanical ventilation, major bleeding (defined as a fall in hemoglobin $\geq 2 \mathrm{~g} / \mathrm{dL}$ or transfusion of $\geq 2$ packed red blood cells units), and time to discharge.

\section{Statistics}

Continuous variables were tested for normality using histograms, quantile-quantile plots, and the Shapiro-Wilk test. Normally distributed continuous variables were compared using the Student's $t$ test and are presented as mean \pm standard deviation (SD). Non-normally distributed continuous variables were compared using the Mann-Whitney test, and are expressed as median and interquartile range. Categorial variables were compared using the Chi-square test and Fisher's exact test, and are presented as numbers and percentiles. In addition to the analysis described above, baseline characteristics were also compared using the standardized mean difference test to evaluate the magnitude of the betweengroup differences, with values $<0.2,0.3-0.5$, and $>0.8$ corresponding to a small, medium, and large effect size, respectively. Comparison of mean and median echocardiographic parameters to reference values was done using the one-sample $t$ test and the Wilcoxon rank-sum test, respectively. Clinical outcomes of the two peaks were illustrated using the Kaplan-Meier model and compared using the logrank test. Adjustment for potential undetected confounders was performed using a multivariate Cox regression model that included age, gender, disease severity, and presence of three or more comorbidities. For all calculations, a $p$ value lower than 0.05 was considered significant. All statistical analysis was performed using SPSS v25. 


\section{Results}

\section{Patient population}

Between March 21 and July 16, 2020, 324 COVID-19 patients were admitted to the Tel Aviv Medical Center. Thirty patients were excluded as they were asymptomatic carriers admitted for either unrelated medical reasons or social arrangements (were unable to self-quarantine). Twenty-seven patients were excluded as they were discharged within less than $24 \mathrm{~h}$ from admission. Nine patients were excluded, since they were designated to palliative care only. Hence, the final study cohort included 258 patients, of which 131 admitted during the first peak and 127 during the second. Mean age was $63 \pm 18$ years and $60.6 \%$ were males. Forty-seven percent of patients had at least three comorbidities, hypertension being the most frequent (50\%), followed by diabetes mellitus (27\%) and obesity (20\%). Mean LVEF was $58 \pm 7 \%$ and $94 \%$ had LVEF $\geq 50 \%$. Right ventricle was dilated in approximately $40 \%$ of patients. Echocardiographic characteristics and reference values are summarized in Table S4. The median lung ultrasound score was 14 (7-19). The median SOFA score was similar in both peaks. Compared to patients that survived, patients that died had a higher median SOFA score in both the first and second peaks (first peak: 1 [0-2] vs 3.5 [2-8.25], $p<0.001$; second peak: 1 [0-2] vs 5 [3-7], $p<0.001)$, whereas no between-peaks difference was found in the median SOFA score of patients that died (first peak: 3.5 [2-8.25], second peak: $5[3-7] ; p=0.4$ ) and in those who survived (first peak: 1 [0-2], second peak: 1 [0-2], $p=0.66$ ). Median COVID-19 MEWS was 3 (1-6). According to the NIH definition of disease severity, $47 \%$ presented with a mild disease, $19 \%$ with a moderate disease, $17 \%$ with a severe disease, and $17 \%$ with a critical disease. Overall, no major differences were noted between patients of the first and second peaks. Patients' baseline characteristics are presented in Table 1.

\section{COVID-19-directed treatment}

COVID-19-directed therapies of patients in the first and second peaks differed considerably. Hydroxychloroquine was the most frequently used drug in the first peak, administrated to $47 \%$ of patients, but the least used drug in the second peak, given to none. Dexamethasone, tocilizumab, remdesivir, and convalescent plasma therapy (CPT) were used significantly more in the second peak $(65 \%$ vs $14 \%$, $p<0.001 ; 39 \%$ vs $15 \%, p<0.001 ; 4.7 \%$ vs $0 \%, p=0.014$ and $43 \%$ vs $8 \%, p<0.001$ respectively). Treatment with full-dose anticoagulation, mostly in the form of lowmolecular-weight heparin, was more frequent during the second peak ( $55 \%$ vs $22 \%$; $p<0.001)$, whereas prophylactic anticoagulation was more common during the first peak (20\% vs $38 \%$; $p=0.001$ ) (Fig. 1a). While no difference was noted in the rate of untreated patients during the second peak, the frequency of treatment with 1-2 COVID-19-directed drugs decreased ( $48 \%$ vs $23 \% ; p<0.001$ ), whereas the frequency of treatment with a combination of 3-4 COVID-19-directed drugs increased significantly (11\% vs $47 \%$; $p<0.001$ ) (Fig. 1b). Among patients with severe-tocritical disease, treatment with dexamethasone, full-dose anticoagulation, tocilizumab, remdesivir, and CPT was significantly more frequent in second peak than in those admitted during the first peak $(93 \%$ vs $20 \%, p<0.001$; $80 \%$ vs $36 \%, p<0.001 ; 76 \%$ vs $20 \%, p<0.001 ; 9.8 \%$ vs $0 \%, p=0.045$ and $58 \%$ vs $15 \%, p<0.001$ ) (Fig. 2a). In the same group of patients, treatment regimens combining 3-4 COVID-19-directed drugs were more frequent in the second peak ( $78 \%$ vs $17 \%, p<0.001)$ (Fig. 2b). In patients with mild-to-moderate disease, apart from tocilizumab and remdesivir, all other COVID-19-directed drugs, as well as regimens of 3-4 drugs, were more common during the second peak (Figures S2 and S3).

\section{Clinical outcomes}

Compared to patients admitted during the first peak, second peak patients had a significantly lower 30-day mortality rate $(10.2 \%$ vs $19.8 \%, p=0.028)$ and lower 30 -day invasive mechanical ventilation rate $(8.8 \%$ vs $19.3 \%, p=0.015$ ) (Fig. 3a,b, respectively). Multivariate Cox regression model adjusting for age, gender, disease severity, and multiple comorbidities showed second peak patients had lower risk for both 30-day mortality and 30-day invasive mechanical ventilation (HR 0.39, 95\% CI $0.19-0.79 ; p=0.009$ and HR $0.29,95 \%$ CI $0.13-0.64$; $p=0.002$, respectively) (Table 2 ). While no significant difference was noted in the rate of non-invasive ventilation between the first and second peaks $(10.8 \%$ vs $15.3 \%$; $p=0.337$ ), the adjusted risk for non-invasive ventilation was significantly higher for patients admitted during the second peak (HR 2.61, 95\% CI 1.24-5.5; $p=0.012$ ), and the median time to intubation was longer in the second peak (4 days [4-11.5] vs. 1 day $[0-3] ; p<0.001$ ). No difference was noted in the rate of major bleeding between the first and second peaks $(2.3 \%$ vs $1.6 \%, p=1)$. Finally, time to discharge was significantly shorter for patients admitted during the second peak (4 days [3-8] vs. 6 days [3-13]; $p=0.002$ ). 
Table 1 COVID-19 modified early waring score ranges from 0 to 23; Lung ultrasound score ranges from 0 (normal lung) to 36 (diffuse bilateral lung consolidations); Sequential organ failure assess- ment score ranges from 0 to 24; SMD: standardized mean difference, values $<0.2,0.3-0.5$, and $>0.8$ correspond to a small, medium, and large effect size, respectively

\begin{tabular}{|c|c|c|c|c|c|}
\hline Parameter & All $N=258$ & 1 st wave $N=131$ & 2nd wave $N=127$ & SMD & $p$ \\
\hline \multicolumn{6}{|l|}{ Demographics } \\
\hline Age, mean \pm SD (years) & $63.5 \pm 18.3$ & $65.2 \pm 17.8$ & $62.1 \pm 18.5$ & 0.17 & 0.162 \\
\hline Male, $n(\%)$ & $157(60.5)$ & $80(61.1)$ & $76(59.8)$ & 0.02 & 0.899 \\
\hline Body mass index, mean $\pm \mathrm{SD}\left(\mathrm{kg} / \mathrm{m}^{2}\right)$ & $27.2 \pm 5.7$ & $26.76 \pm 5.8$ & $27.7 \pm 5.6$ & 0.16 & 0.23 \\
\hline \multicolumn{6}{|l|}{ Medical history } \\
\hline Ischemic heart disease, $n(\%)$ & $40(15.4)$ & $22(16.8)$ & $18(14.2)$ & 0.05 & 0.608 \\
\hline Congestive heart failure, $n(\%)$ & $27(10.4)$ & $13(9.9)$ & $14(11)$ & 0.02 & 0.84 \\
\hline S/P coronary artery bypass graft, $n(\%)$ & $11(4.2)$ & $8(6.1)$ & $3(2.4)$ & 0.14 & 0.217 \\
\hline Atrial fibrillation/flutter, $n(\%)$ & $43(16.6)$ & $23(17.6)$ & $20(15.7)$ & 0.04 & 0.74 \\
\hline Transient ischemic attack/stroke, $n(\%)$ & $26(10)$ & $14(10.7)$ & $12(9.5)$ & 0.03 & 0.837 \\
\hline Peripheral artery disease, $n(\%)$ & $8(3.1)$ & $4(3.1)$ & $4(3.1)$ & 0 & 1 \\
\hline Chronic obstructive pulmonary disease, $n(\%)$ & $14(5.4)$ & $7(5.3)$ & $7(5.5)$ & $<0.01$ & 1 \\
\hline Asthma, $n(\%)$ & $14(5.4)$ & $7(5.3)$ & $7(5.5)$ & $<0.01$ & 1 \\
\hline Chronic kidney disease, $n(\%)$ & $29(11.2)$ & $13(9.9)$ & $16(12.6)$ & 0.07 & 0.557 \\
\hline Diabetes mellitus, $n(\%)$ & $69(26.6)$ & $39(29.8)$ & $30(23.6)$ & 0.11 & 0.325 \\
\hline Smoking, $n(\%)$ & $27(10.4)$ & $17(13)$ & $10(7.9)$ & 0.13 & 0.224 \\
\hline Hypertension, $n(\%)$ & $129(49.8)$ & $74(56.5)$ & $55(43.3)$ & 0.21 & 0.046 \\
\hline Obesity, $n(\%)$ & $53(20.5)$ & $27(20.9)$ & $26(27.1)$ & 0.16 & 0.341 \\
\hline Malignancy, $n(\%)$ & $13(5)$ & $6(4.6)$ & $7(5.5)$ & 0.03 & 0.782 \\
\hline Comorbidities $\geq 3, n(\%)$ & $122(47.1)$ & $62(47.3)$ & $60(47.2)$ & $<0.01$ & 1 \\
\hline \multicolumn{6}{|l|}{ Concurrent medical therapy } \\
\hline Aspirin, $n(\%)$ & $57(22.1)$ & $30(22.9)$ & $27(21.3)$ & 0.03 & 0.766 \\
\hline P2Y12 inhibitor, $n(\%)$ & $14(5.4)$ & $6(4.6)$ & $8(6.3)$ & 0.06 & 0.592 \\
\hline Direct oral anticoagulant, $n(\%)$ & $31(12)$ & $21(16)$ & $10(7.9)$ & 0.19 & 0.055 \\
\hline Angiotensin-converting enzyme, $n(\%)$ & $42(16.3)$ & $22(16.8)$ & $20(15.7)$ & 0.02 & 0.867 \\
\hline Angiotensin receptor blocker, $n(\%)$ & $35(13.6)$ & $20(15.3)$ & $15(11.8)$ & 0.08 & 0.47 \\
\hline Diuretics, $n(\%)$ & $35(13.6)$ & $21(16)$ & $14(11)$ & 0.11 & 0.277 \\
\hline$\beta$-Blocker, $n(\%)$ & $62(24)$ & $34(26)$ & $28(22)$ & 0.07 & 0.471 \\
\hline Systemic corticosteroids, $n(\%)$ & $10(3.9)$ & $4(3.1)$ & $6(4.7)$ & 0.07 & 0.535 \\
\hline Other anti-inflammatories, $n(\%)$ & $7(2.7)$ & $5(3.8)$ & $2(1.6)$ & 0.1 & 0.447 \\
\hline \multicolumn{6}{|l|}{ Laboratory } \\
\hline Hemoglobin, mean \pm SD $(\mathrm{g} / \mathrm{dL})$ & $13.1 \pm 2$ & $13.1 \pm 2.1$ & $13.2 \pm 1.8$ & 0.05 & 0.472 \\
\hline White blood cells, median (IQR) $\left(10^{3} / \mu \mathrm{L}\right)$ & $6.6(5.1-9.4)$ & $7.1(5.3-10)$ & $6.3(4.7-8.7)$ & 0.36 & 0.012 \\
\hline Platelets, median (IQR) $\left(10^{3} / \mu \mathrm{L}\right)$ & $192(145-244)$ & $200(146-265)$ & $182(145-225)$ & 0.26 & 0.062 \\
\hline Creatinine, median (IQR) (mg/dL) & $0.88(0.73-1.13)$ & $0.89(0.73-1.17)$ & $0.86(0.73-1.06)$ & 0.04 & 0.597 \\
\hline Estimated glomerular filtration rate, mean $\pm \mathrm{SD}\left(\mathrm{mL} / \mathrm{min} / 1.73 \mathrm{~m}^{2}\right)$ & $77.9 \pm 29.2$ & $76.37 \pm 28.83$ & $79.48 \pm 29.62$ & 0.11 & 0.393 \\
\hline Estimated glomerular filtration rate $<60 \mathrm{~mL} / \mathrm{min} / 1.73 \mathrm{~m}^{2}, n(\%)$ & $65(25.2)$ & $35(26.7)$ & $30(23.6)$ & 0.05 & 0.569 \\
\hline Blood urea nitrogen, median (IQR) (mg/dL) & $16(11-24)$ & $18(14-27)$ & $14(10-22)$ & 0.11 & $<0.001$ \\
\hline C-reactive protein, median (IQR) (mg/L) & $51(17-126)$ & $57(18-138)$ & $36(16-107)$ & 0.08 & 0.677 \\
\hline C-reactive protein $>5 \mathrm{mg} / \mathrm{L}, n(\%)$ & $233(90)$ & $115(87.8)$ & $118(92.2)$ & 0.11 & 0.208 \\
\hline Troponin-I, median (IQR) (ng/L) & $7(3-20)$ & $9(4-23)$ & $5(3-13)$ & 0.06 & 0.005 \\
\hline Troponin-I > 28 ng/L, $n(\%)$ & $46(17.8)$ & $27(20.9)$ & $19(15)$ & 0.12 & 0.255 \\
\hline BNP, median (IQR) (pg/mL) & $40(14-116)$ & $40(17-141)$ & $37(13-101)$ & 0.05 & 0.351 \\
\hline $\mathrm{BNP}>80 \mathrm{pg} / \mathrm{mL}, n(\%)$ & $68(26.3)$ & $40(32.8)$ & $28(27.7)$ & 0.09 & 0.466 \\
\hline D-dimer, median (IQR) (mg/L) & $0.81(0.47-1.54)$ & $0.9(0.45-1.56)$ & $0.77(0.5-1.43)$ & 0.19 & 0.954 \\
\hline D-dimer > 0.5 mg/L, $n(\%)$ & $180(69.5)$ & $87(69.6)$ & $93(77.5)$ & 0.14 & 0.193 \\
\hline Fibrinogen, mean \pm SD $(\mathrm{mg} / \mathrm{dL})$ & $520 \pm 157$ & $531 \pm 157$ & $510 \pm 158$ & 0.13 & 0.356 \\
\hline Fibrinogen $>470$ mg/dL, $n(\%)$ & $125(48.3)$ & $62(67.4)$ & $63(57.3)$ & 0.17 & 0.149 \\
\hline
\end{tabular}


Table 1 (continued)

\begin{tabular}{|c|c|c|c|c|c|}
\hline Parameter & All $N=258$ & 1st wave $N=131$ & 2 nd wave $N=127$ & SMD & $p$ \\
\hline Ferritin, median (IQR) (ng/mL) & $391(181-945)$ & $450(176-1260)$ & $365(197-780)$ & 0.29 & 0.193 \\
\hline Ferritin $>163 \mathrm{ng} / \mathrm{mL}, n(\%)$ & $174(67.2)$ & $85(78)$ & $89(78.8)$ & 0.01 & 1 \\
\hline \multicolumn{6}{|l|}{ Lung imaging } \\
\hline Lobar infiltration, $n(\%)$ & $38(14.7 \%)$ & $22(17.1)$ & $16(12.8)$ & 0.09 & 0.382 \\
\hline Bilateral infiltration, $n(\%)$ & $101(39)$ & $53(41.1)$ & $48(38.4)$ & 0.04 & 0.701 \\
\hline Pleural effusion, $n(\%)$ & $27(10.4)$ & $22(17.1)$ & $5(4)$ & 0.08 & 0.001 \\
\hline Hilar congestion, $n(\%)$ & $20(7.7)$ & $12(9.3)$ & $8(6.4)$ & 0.08 & 0.487 \\
\hline Lung ultrasound score, median (IQR) & $14(7-19)$ & $15(7-20)$ & $12(9-18)$ & 0.15 & 0.314 \\
\hline \multicolumn{6}{|l|}{ Clinical evaluation of disease severity } \\
\hline Sequential Organ Failure Assessment score, median (IQR) & $1(0-2)$ & $1(0-2)$ & $1(0-2)$ & 0.11 & 0.289 \\
\hline COVID-19 modified early warning score, median (IQR) & $3(1-6)$ & $4(2-7)$ & $3(0-6)$ & 0.26 & 0.075 \\
\hline \multicolumn{6}{|l|}{ National institute of health COVID-19 severity } \\
\hline Mild disease, $n(\%)$ & $121(46.9)$ & $59(45)$ & $62(48.8)$ & 0.06 & 0.618 \\
\hline Moderate disease, $n(\%)$ & $49(19)$ & $25(19.1)$ & $24(18.9)$ & $<0.01$ & 1 \\
\hline Severe disease, $n(\%)$ & $45(17.4)$ & $21(16)$ & $24(18.9)$ & 0.06 & 0.623 \\
\hline Critical disease, $n(\%)$ & $43(16.7)$ & $26(19.8)$ & $17(13.4)$ & 0.13 & 0.185 \\
\hline
\end{tabular}

\section{Discussion}

The main findings of our study are that compared to the first peak of hospitalized COVID-19 patients in our institution, the 30-day mortality and invasive mechanical ventilation rates were significantly lower during the second peak. Importantly, while no major differences were noted between the two groups of patients in baseline characteristics, laboratory parameters, echocardiographic evaluation, lung ultrasound, and disease severity at presentation, the treatment of the two groups differed considerably. The second peak patients were treated with dexamethasone, full-dose anticoagulation, tocilizumab, remdesivir, CPT, as well as a combination of 3-4 drugs at a considerable higher rate compared to patients admitted during the first peak.

\section{Clinical outcomes}

The reduced 30-day mortality rate of second peak patients seen in our cohort could be attributed to several factors. It could be argued that patients of the second peak are younger or have a milder clinical course of illness. However, this seems unlikely as we show that the two groups did not differ in baseline characteristics, laboratory tests results, as well as cardiac and pulmonary imaging. Additionally, disease severity was evaluated using three different well-standardized scores, none of which showed significant difference between patients of the two peaks. Moreover, multivariate analysis adjusting for possible undetected confounding effects of disease severity, age, gender, and multiple comorbidities showed a $60.2 \%$ risk reduction for 30 -day mortality. Similar observations of reduced mortality rate and reduced adjusted mortality risk of hospitalized COVID-19 over time have been reported by Horwitz et al. [12] and Dennis et al. [13]. The findings presented here are novel as they include treatment regimens of all patients. Importantly, as we further corroborate prior findings that survival of hospitalized COVID-19 patients is improving over time, our observations suggest that COVID-19-directed therapies and therapy combination may play a role.

The reduced rate and adjusted risk reduction for invasive mechanical ventilation during the second peak should be interpreted cautiously, as it is a "softer" endpoint that was subjected to physician's discretion. Importantly, during the first peak, experts recommended early invasive ventilation [14], whereas, toward the second peak, as local and global experience was gained, the benefits of early intubation were questioned $[15,16]$, shifting the pendulum toward a practice favoring late intubation and prolonging non-invasive ventilation. Considering that the adjusted risk for non-invasive ventilation was significantly higher during the second peak, it is reasonable that the decrease in invasive mechanical ventilation was, to certain extent, due to physicians' predilection to prolong non-invasive ventilation and avoid early invasive mechanical ventilation. While it is likely that the reduction in both invasive mechanical ventilation and mortality observed in the second peak is a result of changes in medical treatment, we cannot exclude the possibility that avoiding mechanical ventilation, as a clinical approach, is at least partially accountable for the decrease in mortality during the second peak. The lack of randomization as well as the confounding by the difference in therapy regimens do not allow us to determine a causal relationship between these observations. 
a 70

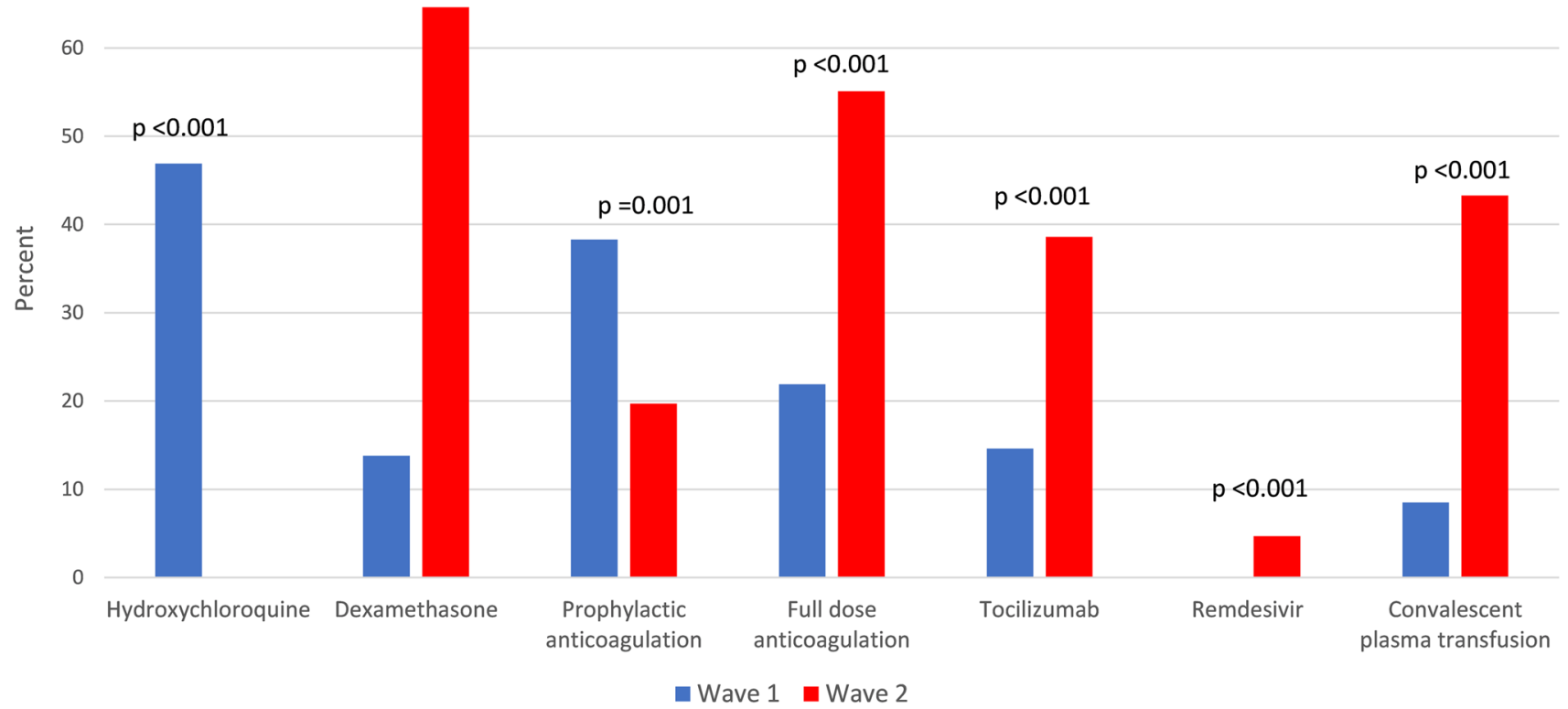

b 60

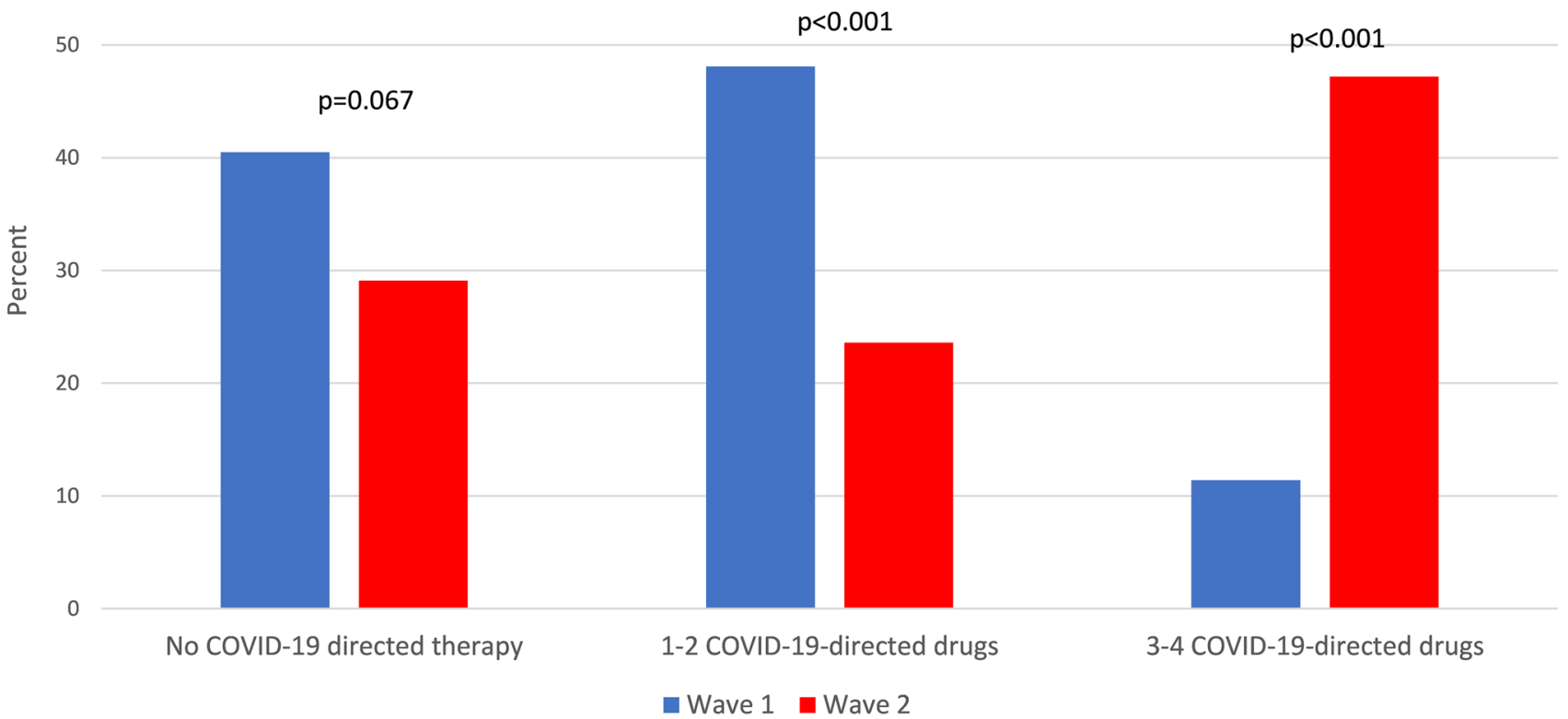

Fig. 1 a COVID-19-directed drugs in the first and second peaks. b COVID-19-directed combination therapies in the first and second peaks

Inspecting the SOFA score within each peak reveals that, in both peaks, not surprisingly, the patients who died were sicker (i.e., had higher SOFA score) compared to those who survived. There was no significant difference in the SOFA score of survivors between the two peaks. There was a non-significant trend toward higher SOFA score of patients who died in the second peak compared to those who died in the first peak. Taken together, these results strengthen the possibility that the change in treatment was the key for the favorable outcomes during the second peak. 


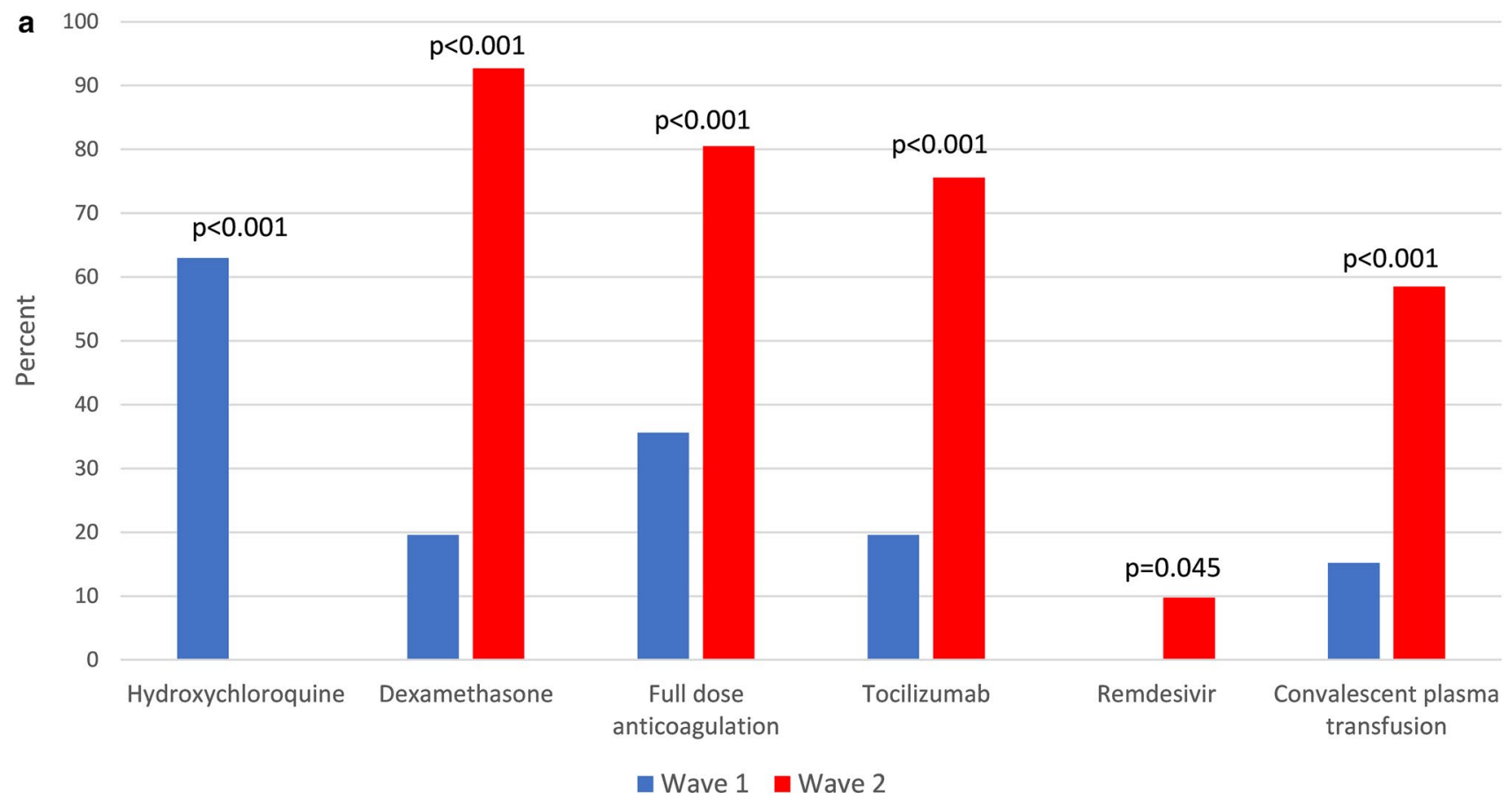

b

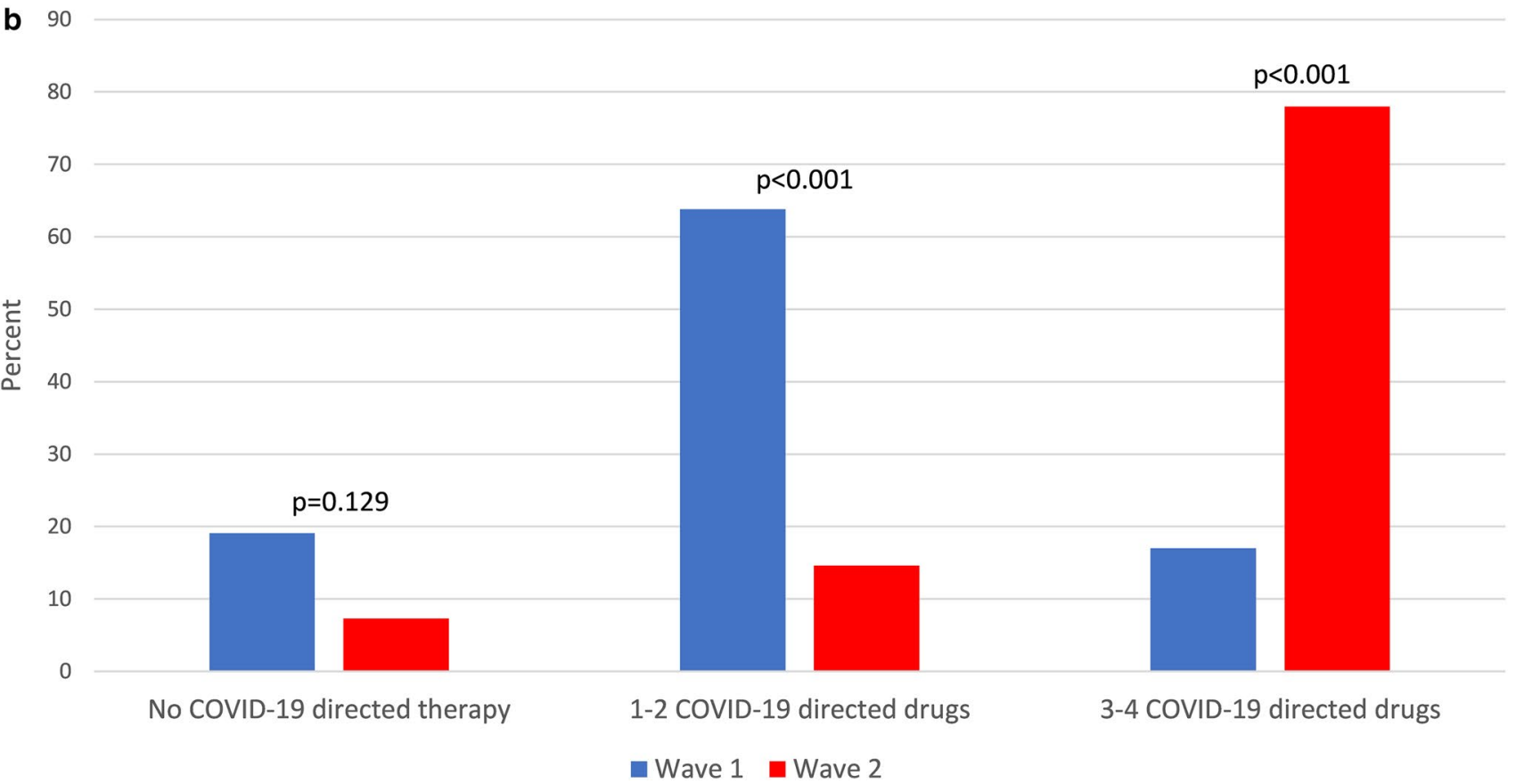

Fig. 2 a First vs second peak COVID-19-directed drugs in patients with severe-to-critical disease. b First vs second peak COVID-19-directed combination therapies in patients with severe-to-critical disease

\section{COVID-19-directed therapies}

Of all the therapies evaluated to date, including those reported in this study, dexamethasone is the only agent that proved to decrease mortality in a large randomized-controlled trial [17]. Although early in the course of the pandemic, the WHO recommended against the routine use of steroids for patients with COVID-19 infection, the preliminary results from the randomizedcontrolled RECOVERY trial [18] published on June 16, 

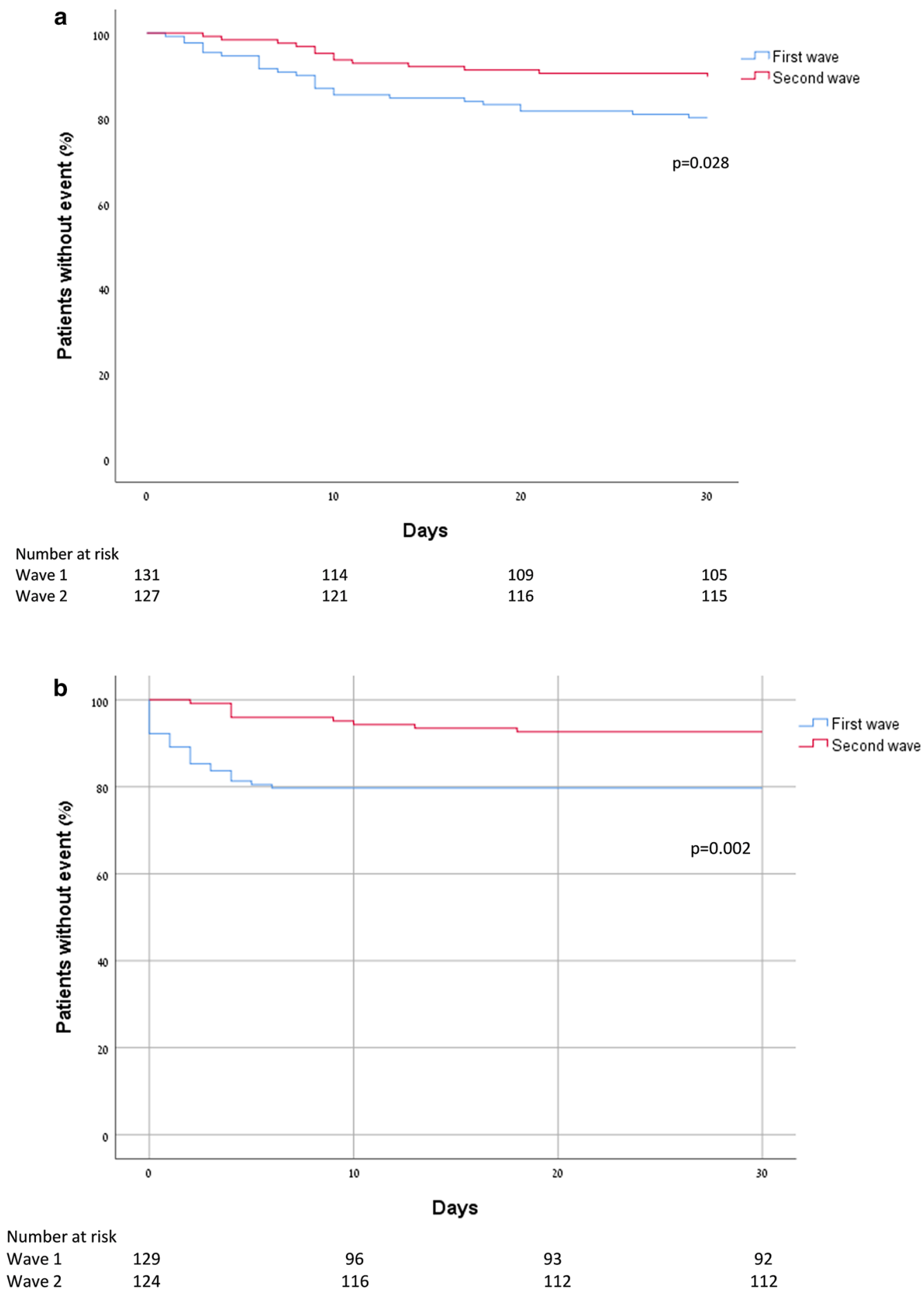

Fig. 3 a Kaplan-Meier curve for 30-day mortality of first and second peaks. b Kaplan-Meier curve for 30-day invasive mechanical ventilation of first and second peaks

2020, established dexamethasone role as the corner-stone in the treatment of hospitalized COVID-19 patients. In our study, dexamethasone use was scarce during the first peak, whereas during the second peak, its use became routine, especially in patients with severe and critical illness, and could account for the favorable outcome observed during the second peak.

To date, data from large randomized-controlled trials concerning the efficacy of anticoagulation in patients with COVID-19 are not available. However, pulmonary 
Table 2 Multivariate Cox regression for the primary outcomes

\begin{tabular}{|c|c|c|c|c|c|c|c|c|}
\hline & \multicolumn{4}{|c|}{ 30-Day mortality } & \multicolumn{4}{|c|}{ 30-Day mechanical ventilation } \\
\hline & \multirow[t]{2}{*}{ HR } & \multicolumn{2}{|l|}{$95 \% \mathrm{CI}$} & \multirow[t]{2}{*}{$p$} & \multirow[t]{2}{*}{ HR } & \multicolumn{2}{|l|}{$95 \% \mathrm{CI}$} & \multirow[t]{2}{*}{$p$} \\
\hline & & Lower limit & Upper limit & & & Lower limit & Upper limit & \\
\hline Second wave & 0.398 & 0.199 & 0.797 & 0.009 & 0.296 & 0.136 & 0.642 & 0.002 \\
\hline Age & 1.061 & 1.028 & 1.095 & $<0.001$ & 1.007 & 0.982 & 1.033 & 0.563 \\
\hline Gender & 0.608 & 0.304 & 1.216 & 0.159 & 0.549 & 0.257 & 1.170 & 0.12 \\
\hline Mild disease & \multicolumn{4}{|c|}{ Reference } & \multicolumn{3}{|c|}{ Reference } & \\
\hline Moderate disease & 0.918 & 0.241 & 3.492 & 0.9 & 1.132 & 0.206 & 6.231 & 0.886 \\
\hline Severe disease & 1.838 & 0.693 & 4.878 & 0.221 & 6.215 & 1.904 & 20.284 & 0.002 \\
\hline Critical disease & 7.489 & 3.187 & 15.599 & $<0.001$ & 20.855 & 6.892 & 63.106 & $<0.001$ \\
\hline Comorbidities $\geq 3$ & 3.639 & 1.304 & 10.156 & 0.014 & 2.03 & 0.881 & 4.678 & 0.096 \\
\hline
\end{tabular}

$H R$ hazard ratio, $C I$ confidence interval post-mortem findings of platelet-fibrin thrombi in small arterial vessels $[19,20]$, as well as the characteristic laboratory coagulation abnormalities of COVID-19 patients [21], suggest that coagulopathy plays a key role in the pathomechanism of the disease and in the respiratory failure associated with severe illness. In our previous study [22] that included the first 100 hospitalized patients of the first peak, we reported that all patients that developed popliteal and femoral deep vein thrombosis, did so while receiving prophylactic doses of low-molecular-weight heparin. Consequently, a more liberal approach favoring treatment with full-dose anticoagulation was adopted during the second peak. Notably, this change in anticoagulation strategy was not associated with increased major bleeding, suggesting that patient selection for full-dose anticoagulation was well balanced. Whether anticoagulation contributed to the decreased mortality and invasive ventilation rates observed during the second peak could not be determined from our study, and randomized-controlled trials evaluating its direct effect are ongoing.

Remdesivir, an inhibitor of viral RNA polymerase, was regarded as a potential therapeutic agent as it showed to inhibit SARS-Cov-2 in vitro [23]. However, the largest randomized-controlled trial held to date [24] reported that although treatment with remdesivir was associated with a shorter time to recovery, it did not decrease mortality compared to placebo. In our study, remdesivir became available shortly prior to the end of the study period, and only six patients admitted during the second peak received it. Thus, the impact of remdesivir on the outcomes reported in our study and its use in combination with other drugs should be considered with caution.

Hydroxychloroquine, initially regarded as a promising therapeutic agent [25], was authorized for emergency use by the Food and Drug and Administration (FDA) [26] only to be revoked shortly thereafter [27], as data raising safety concerns and questioning its efficacy became available [28,
29]. In our cohort, hydroxychloroquine was withdrawn as of April 2, 2020, almost 2 months prior to its revocation by the FDA. Whether the decreased mortality observed in patients of the second peak is, at least partially, due to the exclusion of hydroxychloroquine, remains unknown.

Tocilizumab, a recombinant humanized anti IL-6 monoclonal antibody approved for the treatment of cytokine release syndrome [1] was evaluated in a randomized-controlled trial [30] and was not found to be effective in preventing intubation or death. Importantly, none of the patients in the tocilizumab group received concomitant treatment with dexamethasone, whereas in our study, 49 patients admitted during the second peak were treated with tocilizumab; of those, $98 \%$ received concomitant treatment with dexamethasone.

The therapeutic benefits of CPT have been known for over a century [31], and open-label, non-randomized trials have found it to be efficacious in previous epidemics [32-34]. However, two recently published randomized-controlled trials that evaluated CPT in COVID-19 patient reported conflicting results $[35,36]$, and the role of CPT remains unclear.

The COVID-19 pandemic has far-reaching consequences on health care systems and their ability to contain the morbidity burden while at the same time treating nonCOVID-19 patients. While the availability of a vaccine in such a short time frame is unprecedented and nothing short of a monumental achievement for the scientific community [37], it is probable that COVID-19 will continue to challenge health care systems globally, and it is imperative to continue the search for the optimal treatment for those who will be infected in the future.

Our study has several limitations. It is a single center, retrospective observational study, and a selection bias cannot be excluded. Due to the observational nature of our study and lack of randomization into different treatment groups conclusions regarding individual COVID-19-directed therapies cannot be made, as most patients, especially those with 
a severe and critical disease, received combination therapy. The reduced 30-day mortality and need for ventilation during the second peak could potentially be due to factors not considered in this study.

\section{Conclusions}

Compared to the first peak of hospitalized COVID-19 patients, the 30-day mortality and invasive mechanical ventilation rates as well as the adjusted risks were significantly lower during the second peak. Change in treatment strategies and gain of clinical experience may be responsible for the favorable outcomes. A classic Hebrew phrase says: "All peaks rise in succession and break in their turn, as does man" [38] and Shakespeare wrote: "Like as the waves make toward the pebbled shore, So do our minutes hasten to their end; Each changing place with that which goes before, In sequent toil all forwards do contend." The unique and humbling experience of practicing medicine during a large-scale pandemic, while implementing new treatment strategies in an ever-changing environment, has taught us to never break down, and always pursue better outcomes for our patients.

Supplementary Information The online version contains supplementary material available at https://doi.org/10.1007/s11739-021-02711-1.

Funding None.

\section{Declarations}

Conflict of interests The authors declare that they have no conflict of interest.

Human and animal rights statement The study was approved by the Tel Aviv medical center ethics committee (IRB number 0196-20-TLV).

Informed consent For this retrospective study- formal consent is not required.

\section{References}

1. Huang C, Wang Y, Li X et al (2020) Clinical features of patients infected with 2019 novel coronavirus in Wuhan, China. Lancet. https://doi.org/10.1016/S0140-6736(20)30183-5

2. Li X, Wang W, Zhao X et al (2020) Transmission dynamics and evolutionary history of 2019-nCoV. J Med Virol. https://doi.org/ 10.1002/jmv. 25701

3. Cucinotta D, Vanelli M (2020) WHO declares COVID-19 a Pandemic. Acta Biomed 91(1):157-160. https://doi.org/10.23750/ abm.v91i1.9397

4. European Centre for Disease Prevention and Control An agency of the European Union (2020). https://www.ecdc.europa.eu/en/ publications-data/download-todays-data-geographic-distributioncovid-19-cases-worldwide. Accessed 17 Aug 2020
5. Liao X, Wang B, Kang Y (2020) Novel coronavirus infection during the 2019-2020 epidemic: preparing intensive care unitsthe experience in Sichuan Province, China. Intensive Care Med. https://doi.org/10.1007/s00134-020-05954-2

6. Lambden S, Laterre PF, Levy MM, Francois B (2019) The SOFA score-development, utility and challenges of accurate assessment in clinical trials. Crit Care 23(1):374. https://doi.org/10.1186/ s13054-019-2663-7

7. (2020) COVID-19 Treatment Guidelines Panel. Coronavirus Disease 2019 (COVID-19) Treatment Guidelines. National Institutes of Health. https://www.covid19treatmentguidelines.nih.gov/. Accessed 1 Aug 2020

8. Kirkpatrick JN, Mitchell C, Taub C et al (2020) ASE statement on protection of patients and echocardiography service providers during the 2019 novel coronavirus outbreak. J Am Coll Cardiol 75:3078-3084. https://doi.org/10.1016/j.jacc.2020.04.002

9. Lang RM, Badano LP, Mor-Avi V et al (2015) Recommendations for cardiac chamber quantification by echocardiography in adults: an update from the American Society of Echocardiography and the European Association of Cardiovascular Imaging. J Am Soc Echocardiogr 28:1-39.e14. https://doi.org/10.1016/j.echo.2014. 10.003

10. Topilsky Y, Khanna AD, Oh JK et al (2011) Preoperative factors associated with adverse outcome after tricuspid valve replacement. Circulation 123:1929-1939. https://doi.org/10.1161/CIRCU LATIONAHA.110.991018

11. Lichter Y, Topilsky Y, Taieb P et al (2020) Lung ultrasound predicts clinical course and outcomes in COVID-19 patients. Intensive Care Med 46:1873-1883. https://doi.org/10.1007/ s00134-020-06212-1

12. Horwitz LI, Jones SA, Cerfolio RJ et al (2020) Trends in COVID19 risk-adjusted mortality rates. J Hosp Med. https://doi.org/10. $12788 / \mathrm{jhm} .3552$

13. Dennis JM, McGovern AP, Vollmer SJ, Mateen BA (2020) Improving survival of critical care patients with coronavirus disease 2019 in England. Crit Care Med. https://doi.org/10.1097/ CCM.0000000000004747

14. Marini JJ, Gattinoni L (2020) Management of COVID-19 respiratory distress. JAMA 323:2329. https://doi.org/10.1001/jama.2020. 6825

15. Tobin MJ, Laghi F, Jubran A (2020) Caution about early intubation and mechanical ventilation in COVID-19. Ann Intensive Care 10:78. https://doi.org/10.1186/s13613-020-00692-6

16. Duca A, Memaj I, Zanardi F et al (2020) Severity of respiratory failure and outcome of patients needing a ventilatory support in the Emergency Department during Italian novel coronavirus SARS-CoV2 outbreak: preliminary data on the role of Helmet CPAP and Non-Invasive Positive Pressure Ventilation. EClinicalMedicine 24:100419. https://doi.org/10.1016/j.eclinm.2020. 100419

17. RECOVERY Collaborative Group, Horby P, Lim WS et al (2020) Dexamethasone in hospitalized patients with COVID-19-preliminary report. N Engl J Med. https://doi.org/10.1056/NEJMo a2021436

18. Statement from the Chief Investigators of the Randomised Evaluation of COVid-19 thERapY (RECOVERY) Trial on dexamethasone (2020) Low-cost dexamethasone reduces death by up to one third in hospitalized patients with severe respiratory complications of COVID-19. In: 16 June. https://www.recoverytrial.net/news/ low-cost-dexamethasone-reduces-death-by-up-to-one-third-inhospitalized-patients-with-severe-respiratory-complications-ofcovid-19. Accessed 14 Nov 2020

19. Ackermann M, Verleden SE, Kuehnel M et al (2020) Pulmonary vascular endothelialitis, thrombosis, and angiogenesis in COVID19. N Engl J Med 383:120-128. https://doi.org/10.1056/NEJMo a2015432 
20. Carsana L, Sonzogni A, Nasr A et al (2020) Pulmonary post-mortem findings in a series of COVID-19 cases from northern Italy: a two-center descriptive study. Lancet Infect Dis 20:1135-1140. https://doi.org/10.1016/S1473-3099(20)30434-5

21. Bikdeli B, Madhavan M, Jimenez D et al (2020) COVID-19 and thrombotic or thromboembolic disease: implications for prevention, antithrombotic therapy, and follow-up. J Am Coll Cardiol 75:2950-2973. https://doi.org/10.1016/j.jacc.2020.04.031

22. Szekely Y, Lichter Y, Taieb P et al (2020) Spectrum of cardiac manifestations in COVID-19. Circulation 142:342-353. https:// doi.org/10.1161/CIRCULATIONAHA.120.047971

23. Wang M, Cao R, Zhang L et al (2020) Remdesivir and chloroquine effectively inhibit the recently emerged novel coronavirus (2019-nCoV) in vitro. Cell Res 30:269-271. https://doi.org/10. 1038/s41422-020-0282-0

24. Beigel JH, Tomashek KM, Dodd LE et al (2020) Remdesivir for the treatment of COVID-19-final report. N Engl J Med 383:1813-1826. https://doi.org/10.1056/NEJMoa2007764

25. Gautret P, Lagier J-C, Parola P et al (2020) Hydroxychloroquine and azithromycin as a treatment of COVID-19: results of an open-label non-randomized clinical trial. Int J Antimicrob Agents 56:105949. https://doi.org/10.1016/j.ijantimicag.2020.105949

26. Hinton Denise M (2020) Request for emergency use authorization for use of chloroquine phosphate or hydroxychloroquine sulfate supplied from the strategic national stockpile for treatment of 2019 coronavirus disease. https://www.fda.gov/media/136534/ download. Accessed 1 Aug 2020

27. Coronavirus (COVID-19) Update: FDA Revokes Emergency Use Authorization for Chloroquine and Hydroxychloroquine. In: 2020. https://www.fda.gov/news-events/press-announcements/coron avirus-covid-19-update-fda-revokes-emergency-use-authorizat ion-chloroquine-and. Accessed 1 Aug 2020

28. Hraiech S, Bourenne J, Kuteifan K et al (2020) Lack of viral clearance by the combination of hydroxychloroquine and azithromycin or lopinavir and ritonavir in SARS-CoV-2-related acute respiratory distress syndrome. Ann Intensive Care 10:63. https://doi.org/ 10.1186/s13613-020-00678-4
29. Szekely Y, Lichter Y, Shrkihe BA et al (2020) Chloroquineinduced torsade de pointes in a COVID-19 patient. Heart Rhythm. https://doi.org/10.1016/j.hrthm.2020.04.046

30. Stone JH, Frigault MJ, Serling-Boyd NJ et al (2020) Efficacy of tocilizumab in patients hospitalized with COVID-19. N Engl J Med. https://doi.org/10.1056/NEJMoa2028836

31. Casadevall A, Scharff MD (1995) Return to the past: the case for antibody-based therapies in infectious diseases. Clin Infect Dis 21:150-161. https://doi.org/10.1093/clinids/21.1.150

32. Zhou B, Zhong N, Guan Y (2007) Treatment with convalescent plasma for influenza A (H5N1) infection. N Engl J Med 357:1450-1451. https://doi.org/10.1056/NEJMc070359

33. Hung IF, To KK, Lee C-K et al (2011) Convalescent plasma treatment reduced mortality in patients with severe pandemic influenza A (H1N1) 2009 virus infection. Clin Infect Dis 52:447-456. https://doi.org/10.1093/cid/ciq106

34. Cheng Y, Wong R, Soo YOY et al (2005) Use of convalescent plasma therapy in SARS patients in Hong Kong. Eur $\mathbf{J}$ Clin Microbiol Infect Dis 24:44-46. https://doi.org/10.1007/ s10096-004-1271-9

35. Simonovich VA, Pratx LDB, Scibona P et al (2020) A randomized trial of convalescent plasma in COVID-19 severe pneumonia. $\mathrm{N}$ Engl J Med. https://doi.org/10.1056/NEJMoa2031304

36. Libster R, Marc GP, Wappner D et al (2021) Early high-titer plasma therapy to prevent severe COVID-19 in older adults. $\mathrm{N}$ Engl J Med. https://doi.org/10.1056/NEJMoa2033700

37. Polack FP, Thomas SJ, Kitchin N et al (2020) Safety and efficacy of the BNT162b2 mRNA COVID-19 vaccine. N Engl J Med 383:2603-2615. https://doi.org/10.1056/NEJMoa2034577

38. Shitrit M (2016) Sometimes. https://www.youtube.com/watch?v= TxeYFbC1HWM. Accessed 18 Aug 2020

Publisher's Note Springer Nature remains neutral with regard to jurisdictional claims in published maps and institutional affiliations. 